\title{
LUMINOSITY OF $\delta$ SCUTI STARS AFTER HIPPARCOS SATELLITE
}

\author{
E. ANTONELLO, L. MANTEGAZZA, E. PORETTI \\ Osservatorio Astronomico di Brera, Via E. Bianchi 46, 22055 Merate, Italy
}

The absolute magnitudes of $\delta$ Scuti stars derived from parallaxes measured by the Hipparcos satellite were compared with the previous estimates based on photometric uvby $\beta$ indices, and significant differences were found which are related to photometric effects of metallicity and rotational velocity. A reliable calibration of $M_{V}$ in terms of the photometric indices shall include an estimate of these effects. It is important also to take into account the possible presence of unresolved close companions in order to fully exploit the accuracy of $M_{V}$ of nearby stars derived from the trigonometric parallaxes.

The $M_{V}$ of few bright SX Phe stars support the period-luminosity relation obtained with ground based observations of globular clusters, while it does not seem to confirm the empirical dependence of this relation on the metallicity.

Some high amplitude $\delta$ Scuti stars with intermediate or normal metallicity and small and uncertain parallax have apparently a very low luminosity. Simulations of $M_{V}$ determinations from observed parallaxes based on the discussion of observational errors by Lutz and Kelker (1973) have shown that the low luminosity could be an effect related to these errors.

\section{References}

Lutz, T.E. and Kelker, D.H. (1973) Publ.Astr.Soc.Pacific, Vol. no. 85, pp. 573-578.

\section{THE DISTANCES AND ABSOLUTE MAGNITUDES OF SOME WELL-KNOWN RED VARIABLES}

\author{
ROBERT F. WING \\ Astronomy Department, Ohio State University, Columbus OH, USA
}

Distances and absolute magnitudes have been obtained from an early distribution of Hipparcos data for a number of well-known late-type variables. These data were requested in 1981 in order to ensure that these important stars were not excluded from the Input Catalogue on account of their variability or very red color.

Measurements were requested for 20 Mira variables, and 14 of these were successfully observed. Of these, the nearest to the Sun were found to be R Leo (at $102 \mathrm{pc}$ ), $\chi$ Cyg (106), R Cas (107), $\mathrm{R}$ Car (128), T Cep (210), and R Aql (211), with formal errors ranging from \pm 12 to $\pm 57 \mathrm{pc}$. Mira itself was not considered because the presence of a companion causes an 11-month wobble in its photocenter which is likely to lead to a spurious parallax. The absolute visual magnitudes of Miras at mean maximum range approximately from -2.5 to +2.0 with a clear dependence on spectral type (cooler stars being fainter). This trend is reversed for absolute magnitudes in the near infrared.

Results are also given for 10 small-amplitude variables of late type. The nearest of these are $\mathrm{L}_{2}$ Pup (M5 III, $61 \mathrm{pc}$ ) and R. Dor (M8e, $62 \mathrm{pc}$ ). The symbiotic star CH Cyg lies at a rather uncertain distance of $268 \pm 65 \mathrm{pc}$.

Data were obtained for 23 carbon stars, including the brightest representatives of each of several subgroups. The nearest of these was found to be the SRb variable U Hya, at $162 \pm 20 \mathrm{pc}$. The stars 19 Psc, Y CVn, W Ori, and HD 201626 were all found to be somewhat more than $200 \mathrm{pc}$ away. 